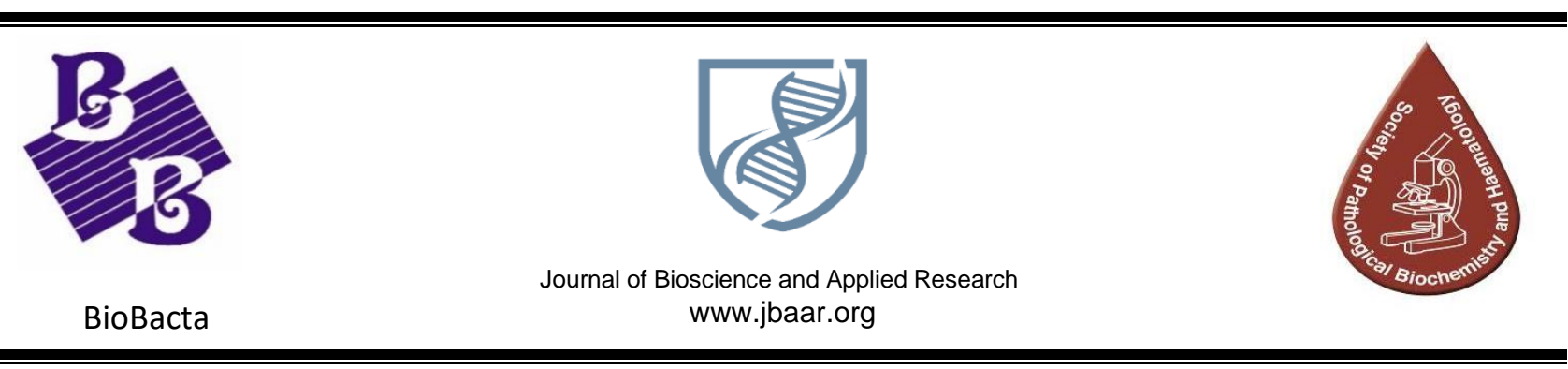

\title{
Evaluation of diagnostic performances of leptin and vitamin $D$ for colorectal cancer diagnosis and follow-up
}

\author{
Manar S. Fouda ${ }^{1}$, Sara A. Mekkawy ${ }^{2}$, Mariam T. Ghabour ${ }^{2}$, Radwa E. Othman², Nayera A. \\ Ahmed $^{2}$, Nour A. Habbib ${ }^{2}$, Salsabeel A.Elkholey ${ }^{2}$, Rahma M.Soliman², Maria C. Fouad ${ }^{2}$, \\ Ellen Y.Ayad², Mayar A. Shaqran², Mariam I Mohamed², Rokaia M. Aljarwani', Khaled \\ Aboul-Enein ${ }^{3}$, Mohamed M. Omran ${ }^{1 *}$ \\ ${ }^{1}$ Chemistry Department, Faculty of Science, Helwan University, Ain Helwan, Cairo, Egypt \\ ${ }^{2}$ Molecular Biotechnology Program, Faculty of Science, Helwan University, Ain Helwan, Cairo, Egypt \\ ${ }^{3}$ Clinical Pathology Department, National Cancer Institute, Cairo University, Giza, Egypt.

\section{*Correspondence:} \\ Mohamed Mostafa Omran, Chemistry Department, Faculty of Science, Helwan University, Ain Helwan, 11795, Cairo, \\ Egypt, E-mail: drmmomran@science.helwan.edu.eg; drmmomran@yahoo.com
}

DOI: $10.21608 /$ JBAAR. 2021.198090

\begin{abstract}
:
Globally, colorectal cancer (CRC) is one of the most often diagnosed solid tumors, with a significant death and morbidity rate. CRC biomarkers are desperately needed for early detection. Traditional CRC tumor markers do not have the best diagnostic performance. The levels of leptin and vitamin D were evaluated. CRC patients before treatment $(n=16)$, CRC patients after treatment $(n=14)$, and 20 patients with benign tumors were included in this case-control study. ELISA was used to determine the levels of traditional tumor markers (CA19.9 and CEA) as well as candidate markers (leptin and vitamin D). Using area receiver-operating characteristic analysis (AUC), the diagnostic performance of single and combination markers was assessed (ROC). The levels of CEA and CA 19.9 in the three groups studied were not significantly different. Vitamin $\mathrm{D}$ and leptin were significantly decreased $(\mathrm{p}=0.03$ and $\mathrm{p}=0.02$; respectively) in CRC patients than after benign patients. A novel combination, based on the combination of vitamin D and leptin was developed for CRC diagnosis using stepwise multivariate discriminant analysis (MDA). The combination can be represented as = $(4.65$ - vitamin D $((\mathrm{ng} / \mathrm{ml})) \times 0.009+$ Leptin $(\mathrm{ng} / \mathrm{ml}) \times 0.441)$. AUCs were reported when leptin was used as a single biomarker for distinguishing CRC from benign (0.78) and non-treated CRC from treated CRC (0.67). When leptin and vitamin D were combined, the AUCs increased to 0.84 and0.72, respectively. Conclusion: Leptin and vitamin D were shown to be promising diagnostic and follow-up indicators for CRC in our investigation.
\end{abstract}

Keywords: Colorectal cancer (CRC), Leptin, vitamin D, ELISA 


\section{Introduction}

CRC is the world's second most often diagnosed cancer and the $3^{\text {rd }}$ major cause of cancer mortality (Bray et al., 2018). Colorectal cancer is predicted to increase to 25 million new cases globally by 2035 (Arnold et al., 2017), owing to continued development in developed countries. Only highly developed countries exhibit stabilizing and declining patterns. Both have been attributed largely to national screening programs and improved colonoscopy adoption in general, while dietary and lifestyle changes can also play a role (Ouakrim et al., 2015). In comparison, a concerning increase in patients younger than 50 years old who present with colorectal cancer, especially rectal cancer, and left-sided colon cancer, has been observed (Kasi et al., 2019; Wolf et al., 2018). While there may be a connection between genetics, lifestyle, obesity, and environmental factors, the exact causes of this increase are unknown (Dekker et al., 2019).

Vitamin D is a family of steroid hormones that increases calcium, magnesium, and phosphate absorption in the intestine, among other things (Pludowski et al., 2018). The importance of vitamin $\mathrm{D}$ in CRC has piqued researchers' interest. The researchers calculated the quantity of vitamin $\mathrm{D}$ after exposure to ultraviolet radiation. Increased 25hydroxy vitamin $\mathrm{D}(25(\mathrm{OH}) \mathrm{D})$ concentrations in the blood, the most accurate indicator of vitamin $\mathrm{D}$ status in the body, were associated with improved survival in most prospective observational trials that looked at a relationship with prognosis in CRC patients (Fedirko et al., 2012). Cancer cells are thought to absorb and stimulate $25(\mathrm{OH}) \mathrm{D}$, which binds to the vitamin $\mathrm{D}$ receptor to control gene expression and, as a result, tumor pathophysiology (Harten-gerritsen et al., 2015). Several studies examined the relationship between blood 25(OH)D concentrations and CRC patient survival (Hoffmeister \& Brenner, n.d.; Wu et al., 2020).
One theory is that obesity and CRC are associated with the release of many hormones known as adipokines, the most important of which are leptin and adiponectin. Leptin is a $16 \mathrm{kDa}$ peptide cytokine formed exclusively by adipose tissues. Leptin is a hormone that controls food consumption and energy output. Its concentration is inversely proportional to body mass index (BMI), so it is higher in obese people. Leptin has been shown to have a mitogenic, anti-apoptotic, and tumorigenic role on a variety of cancer cell lines, including colorectal in many laboratory studies. Furthermore, leptin and its receptor were shown to be substantially upregulated in CRC tissues relative to normal tissues, and this upregulation was linked to a more advanced tumor phenotype (Mhaidat et al., 2021; Tojek et al., 2019).

\section{Materials and Methods}

Fifty patients were given by the Egyptian National Cancer Institute. Patients with histologically proven adenocarcinoma of the colon or rectum $(n=16)$, CRC patients after treatment $(n=14)$, and 20 patients with benign tumors were divided into three groups. Treatment options included chemotherapy, radiation, concurrent chemo and/or radiotherapy, and surgery. Clinical data from patient's medical records were gathered, including age, gender, tumor histology, stage, and treatment methods. The following items were estimated before treatment: blood exams (CEA, CA19.9), Among the techniques done include computed tomography (CT), chest roentgenogram, abdominal ultrasound screening, and colonoscopy. The study's clinical staging was done utilizing the TNM (tumor, node, and metastasis) colorectal cancer categorization method (Siegel and colleagues, 2014).

\section{Biochemical analyses}

Blood samples were collected using an automated biochemistry analyzer for regular laboratory exams, such as hepatic and kidney biochemical profile tests (Beckman Coulter AU680 chemistry analyzer, USA). An automated hematology analyzer was used to calculate the complete blood count (Sysmex XN 
1000, Japan). According to the manufacturer's instructions, serum CA19.9 and CEA were determined using an ELISA test (ARCHITECT I 1000 SR immunoassay analyzer, Abbott USA).

\section{Determination of Leptin using ELISA}

Leptin is injected into wells that have been precoated with the LEP monoclonal antibody. A total of $50 \mu \mathrm{l}$ of serum samples or standard concentrations ranging from 0.4 to $6.4 \mathrm{ng} / \mathrm{ml}$ were added. The antihuman LEP antibody $(10 \mu \mathrm{l})$ is biotin-conjugated and binds to human LEP. The biotin-conjugated antihuman LEP antibody is bound by streptavidin-HRP $(50 \mu 1)$. During the washing phase after incubation, all unbound Streptavidin-HRP is rinsed away (5 times for 1 minute for each wash). Add $50 \mu 11$ of substrate solution A to each well, followed by $50 \mu 11$ of substrate solution $\mathrm{B}$. At $37^{\circ} \mathrm{C}$, incubate the plate for 10 minutes. The process is stopped by adding a $50 \mu \mathrm{l}$ acidic stop solution and measuring the absorbance at $450 \mathrm{~nm}$. Within 30 minutes after applying the stop solution, determine the optical density (OD value) of each well using a microplate reader (Acculabusa, MR96Microplate Read, Avenue De Lorimier Bureau, Montréal, Canada) set to $450 \mathrm{~nm}$.

\section{Determination of vitamin $D$ using competitive ELISA}

ELISA plate wells coated with an Anti-vitamin D antibody treated with $10 \mu \mathrm{l}$ of vitamin D standards (2.5 $-150 \mathrm{ng} / \mathrm{ml})$ or samples, and $200 \mu$ of vitamin DBiotin conjugate. vitamin D competes with vitamin D in serum, serial standards for a fixed no. of binding sites on the anti-vitamin D antibody. Binded vitamin D-Biotin was identified with $200 \mu$ l StreptavidinHRP after a wash step with $300 \mu$ l washing solution (3 times for 1 minute for each wash) (SA-HRP). As the concentration of vitamin $\mathrm{D}$ in the sample rises, the SA-HRP conjugate immunologically bound to the wells decreases. The wells were then rinsed to eliminate any unbound SA-HRP conjugate (3 times for 1 minute for each wash). After that, a solution of $3,3$ ',5,5'-Tetramethylbenzidine substrate (200 $\mu \mathrm{l})$ was added and incubated for 15 minutes at room temperature, resulting in the production of blue color. The inhibition of color occurring after the addition of the stop solution. The absorbances were measured at $450 \mathrm{~nm}$ with a microplate reader (Acculabusa, MR $\neg 96$ Micro $\neg$ plate Read, Avenue De Lorimier Bureau, Montréal, Canada).

\section{Statistical analyses}

The Statistical Package for the Social Sciences (SPSS) operating system, version15, was used to conduct the statistical analysis (SPSS Inc., Chicago, IL). The mean and standard deviation (SD) were used for parametric data, while the median or interquartile range was used for non-parametric data. To find statistically significant differences, Kruskal-Wallis, ANOVA, and the Student's t-test were employed. The optimal combination was created by combining the most discriminating independent variables using the stepwise logistic regression algorithm. The diagnostic performance of standard and candidate tumor markers was assessed using AUC. Receiveroperating characteristic analysis was used to identify the optimal cutoff thresholds and diagnostic performance (ROC).

\section{Results}

The fundamental clinical characteristics and parameters are shown in Table 1; In this study, 50 patients with CRC (28 men and 22 women), including 16 non-treated CRC and 14 treated CRC, and 20 patients with benign disease (13 men and 7 women) were enrolled. CEA, CA19.9, Vitamin D, and Leptin values in benign individuals, treated CRC, and nontreated CRC are shown in Table 2. There is no significant difference in the levels of routine laboratory parameters between CRC and benign. Traditional markers commonly used in CRC diagnosis, such as CEA and CA19.9, showed no significant differences between the three study groups. Vitamin D and leptin levels were substantially lower $(\mathrm{p}=0.03$ and $\mathrm{p}=0.02$ ) in CRC patients than in benign individuals. Traditional 
indicators CEA and CA19.9, as well as vitamin D and leptin levels, did not change significantly between treated and untreated CRC.

The diagnostic power evaluation of single and combined markers

A novel combination, based on the combination of vitamin D and leptin was developed for CRC diagnosis and follow-up. The combination can be represented as $=(4.65-$ vitamin $\mathrm{D}(\mathrm{IU}) \times 0.009+$ Leptin $(\mathrm{ng} / \mathrm{ml}) \times 0.441)$. The assessment of the preferential power of single or combined markers was performed via ROC analysis to distinguishing CRC patients from benign ones. Table 3 showed that leptin was used as a single marker, it had an extremely preferential AUC of 0.78, which improved to 0.84 when leptin and vitamin $\mathrm{D}$ were combined for distinguishing malignant CRC from benign subjects. Leptin had the highest ability to monitor the treatment $($ AUC $=0.67)$ and combined Leptin and vitamin D increased AUC to 0.72 for discriminating treated CRC patients from non-treated.

Table 1. Clinical data of CRC patients and benign characteristics

\begin{tabular}{ll}
\hline Parameters & Value (\%) \\
No. of patients & 50 \\
Non-treated CRC & $16(32 \%)$ \\
Treated CRC & $14(28 \%)$ \\
Benign & $20(40 \%)$ \\
Mean age, years (range) & $48(28-81)$ \\
Cancer position, no. & \\
Colon & $28(56 \%)$ \\
Rectal & $22(44 \%)$ \\
Tumor size & \\
T1 & $7(14 \%)$ \\
T2 & $20(40 \%)$ \\
T3 & $11(22 \%)$ \\
Unknown & $12(24 \%)$ \\
Tumor grade, no. & \\
G1 & $3(6 \%)$ \\
G2 & $26(52 \%)$ \\
G3 & $10(20 \%)$ \\
Unknown & $11(22 \%)$ \\
Lymph node status, no. & \\
Positive & $8(16 \%)$ \\
Negative & $30(60 \%)$ \\
Unknown & $12(24 \%)$ \\
Metastasis & \\
Positive & $5(10 \%)$ \\
Negative & $33(66 \%)$ \\
Unknown & $12(24 \%)$ \\
Stage, no. & \\
I & $24(48 \%)$ \\
II & $7(14 \%)$ \\
IV & $3(6 \%)$ \\
Unknown & $4(8 \%)$ \\
& $12(24 \%)$ \\
\hline
\end{tabular}


Table 2. levels of routine and candidate markers in studied groups

\begin{tabular}{|c|c|c|c|c|c|c|}
\hline Variables & Benign & $\begin{array}{c}\text { All CRC } \\
\text { patients }\end{array}$ & $\begin{array}{c}\text { Non-treated } \\
\text { CRC }\end{array}$ & $\begin{array}{c}\text { Treated } \\
\text { CRC }\end{array}$ & $\begin{array}{c}\mathbf{P}^{\mathbf{1}} \\
\text { value }\end{array}$ & $\begin{array}{c}\mathbf{P}^{\mathbf{2}} \\
\text { value }\end{array}$ \\
\hline CEA (ng/ml) & $2.2(1.5-5.3)$ & $6.7(2.7-23.5)$ & $5.6(1.1-56)$ & $10(4.7-7.8)$ & 0.16 & 0.43 \\
\hline CA19.9 (u/ml) & $24(4.3-32)$ & $23(10-88)$ & $48(13-306)$ & $22(2.3-83)$ & 0.17 & 0.99 \\
\hline $\begin{array}{c}\text { Vitamin D } \\
(\mathrm{ng} / \mathrm{ml})\end{array}$ & $69.6 \pm 12.1$ & $59.5 \pm 15$ & $54.7 \pm 17.5$ & $62.4 \pm 12.6$ & 0.03 & 0.18 \\
\hline \begin{tabular}{c} 
Leptin $(\mathrm{ng} / \mathrm{ml})$ \\
\hline
\end{tabular} & $2.9 \pm 0.3$ & $2.6 \pm 0.4$ & $2.5 \pm 0.4$ & $2.7 \pm 0.3$ & 0.02 & 0.17 \\
\hline
\end{tabular}

$\mathrm{P}^{1}$ value $=$ benign vs CRC

$\mathrm{P}^{2}$ value $=$ benign vs treated

Table 3. Diagnostic performances of single and combined markers

\begin{tabular}{cccccccc}
\hline Variables & AUC $(95 \%$ CI $)$ & P value & Cut-off & Sensitivity & Specificity & PPV & NPV \\
& & \multicolumn{7}{c}{ CRC vs Benign } \\
CEA & $0.55(0.45-0.65)$ & 0.75 & 2.8 & 60 & 50 & 68 & 55 \\
CA19.9 & $0.60(0.47-0.70)$ & 0.17 & 23.4 & 62 & 58 & 69 & 58 \\
Vit. D & $0.75(0.58-0.92)$ & 0.01 & 58.1 & 62 & 75 & 67 & 71 \\
Leptin & $0.78(0.63-0.94)$ & 0.004 & 1.73 & 75 & 70 & 67 & 78 \\
Vit. D+ Leptin & $0.84(0.71-0.98)$ & 0.001 & 2.87 & 81 & 75 & 72 & 83 \\
& & Non treated CRC vs Non treated CRC & & & \\
CEA & $0.45(0.25-0.55)$ & 0.74 & 2.8 & 54 & 55 & 66 & 48 \\
CA19.9 & $0.55(0.35-0.64)$ & 0.68 & 23.4 & 42 & 50 & 50 & 43 \\
Vit. D & $0.66(0.48-0.75)$ & 0.09 & 58.1 & 57 & 56 & 55 & 53 \\
Leptin & $0.67(0.50-0.78)$ & 0.07 & 1.73 & 58 & 66 & 56 & 55 \\
Vit. D+ Leptin & $0.72(0.53-0.85)$ & 0.04 & 2.87 & 75 & 58 & 68 & 63 \\
\hline
\end{tabular}




\section{Discussion}

CRC is one of the leading causes of death worldwide, so early detection is critical. CEA and CA 19-9 levels are elevated in both malignancies and benign disorders and are not limited to malignancies. Standard markers (CEA and CA19-9) had reduced sensitivity and specificity in distinguishing malignant from benign tumors. Leptin, Vitamin D, CEA, and CA 19-9 were tested for their ability to detect CRC early and distinguished between malignant and benign CRC patients. When comparing patients with CRC to those with benign tumors, we found that leptin and vitamin D levels were substantially lower in CRC patients.

The negative relationship between dietary vitamin D3 consumption or sunlight exposure and human colorectal cancer has been determined to be $50 \%$, which is linked to the inverse relationship between dietary vitamin D3 intake or sunlight exposure and human colorectal cancer (Klampfer, 2014). Increasing vitamin D3 levels have been linked to a decreased risk of colon cancer and polyp recurrence, as well as enhanced overall survival in colon cancer patients, according to many studies.

Several studies have speculated a correlation between elevated leptin levels and colon cancer risk after the discovery of leptin's role as a growth factor for colonic epithelial cancer (Hardwick et al., 2001; Aparicio, 2005). On an inflammatory basis, leptin causes the inflammatory cytokines IL-6, IL-1, and CXCL1 to be secreted in humans, which have all been linked to colon cancer (Padidar et al., 2011). In particular, CXCL1 secretion supports in vitro experiments that show leptin stimulates vascular endothelial growth factor (VEGF) activity by epithelial cells, providing a pathway for tumorassociated angiogenesis and thereby facilitating tumors survival and proliferation (Cascio et al., 2009). Indeed, leptin promotes tumors development and invasion of nearby organs by promoting angiogenesis (Koda et al., 2007).
In this study, the level of vitamin $\mathrm{D}$ was significantly lower in malignant CRC patients than in benign CRC patients and leptin was increased, while CEA and CA 19-9 levels were not significantly different in malignant $\mathrm{CRC}$ patients relative to benign CRC patients. Our results agreed with the previous studies and covered the drawbacks of them as most of them were performed in animal models but our study was performed on patient samples. The AUC of single thymidine kinase 1, CA19-9, CA72-4, and CEA and combined four markers were $0.86,0.74$, 0.75, 0.79, and 0.95, respectively (Ning et al., 2018). Attallah et al. developed a model based on CEA, CA19.9, cytokeratin 1, and mucin-1. The AUC of this model was 0.84 for colon cancer versus benign was higher than each of the individual markers (CA19.9: 0.58; CA19.9: 0.60; cytokeratin 1: 0.75; mucin-1: 0.73). LRG1 was utilized by Fouda et al. to distinguish CRC from healthy(0.87), benign(0.84), and non-treated CRC vs treated CRC (0.82). When LRG1 and SCF were coupled, AUCs increased to0.90,0.84, and0.84, respectively. Sun et al., 2019 used a combination of fibrinogen/pre-Albumin, CEA, and CA199 with AUC of 0.85 for differentiated CRC from benign. In conclusion, the combination of vitamin $\mathrm{D}$ and leptin presented in this study intends to improve early CRC diagnosis and follow-up.

\section{References}

- Ait Ouakrim, D., Pizot, C., Boniol, M., et al. (2015). Trends in colorectal cancer mortality in Europe: retrospective analysis of the WHO mortality database. BMJ, h4970.

- Anchal, Srivastava, Shukla, R. K., Nishant Kumar, Anu Katiyar. (2017). Nanoparticles as Biomarkers and Biosensors. Curr Trends Biomedical Eng \& Biosci. 2017; 9(3): 555762.

- Aparicio, T. (2005). Leptin stimulates the proliferation of human colon cancer cells in vitro but does not promote the growth of colon cancer xenografts in nude mice or intestinal 
tumorigenesis in ApcMin/+ mice. Gut, 54(8), 1136-1145.

- Arnold, M., Sierra, M. S., Laversanne, M., Soerjomataram, I., Jemal, A., Bray, F. (2016). Global patterns and trends in colorectal cancer incidence and mortality. Gut, 66(4), 683691.

- Attallah, A. M., El-Far, M., Ibrahim, A. R., et al. (2018). Clinical value of a diagnostic score for colon cancer based on serum CEA, CA19-9, cytokeratin-1 and mucin-1. Br J Biomed Sci, 75(3):122-127.

- Bosman, F. T., Yan, P. (2014). Molecular pathology of colorectal cancer. Polish Journal of Pathology, 4, 257-266.

- $\quad$ Bray, F., Ferlay, J., Soerjomataram, I., Siegel, R. L., Torre, L. A., Jemal, A. (2018). Global Cancer Statistics 2018: GLOBOCAN Estimates of Incidence and Mortality Worldwide for 36 Cancers in 185 Countries. CA Cancer J Clin, 68(6):394-424.

- Cascio, S., Ferla, R., D’Andrea, A., Gerbino, A., Bazan, V., Surmacz, E., Russo, A. (2009). Expression of angiogenic regulators, VEGF and leptin, is regulated by the EGF/PI3K/STAT3 pathway in colorectal cancer cells. Journal of Cellular Physiology, 221(1), 189-194.

- Dekker, E., Tanis, P. J., Vleugels, J. L. A., Kasi, P. M., Wallace, M. B. (2019). Colorectal cancer. The Lancet, 394(10207), 1467-1480.

- Fedirko, V., Riboli, E., Tjonneland, A., et al. (2012). Prediagnostic 25-Hydroxyvitamin D, VDR and CASR Polymorphisms, and Survival in Patients with Colorectal Cancer in Western European Populations. Cancer Epidemiology Biomarkers \& Prevention, 21(4), 582-593.

- Fouda, M. S., Aljarwani, R. M., Aboul-Enein, K., Omran, M. M. (2021). Diagnostic performances of leucine-rich $\alpha$-2-glycoprotein 1 and stem cell factor for diagnosis and follow-up of colorectal cancer. J Genet Eng Biotechnol, 19(1):17.
- Gröschel, C., Aggarwal, A., Tennakoon, S., et al. (2016). Effect of 1,25-dihydroxyvitamin D3 on the Wnt pathway in non-malignant colonic cells. The Journal of Steroid Biochemistry and Molecular Biology, 155, 224-230.

- Hardwick, J. C. H., Van Den Brink, G. R., Offerhaus, G. J., Van Deventer, S. J. H., Peppelenbosch, M. P. (2001). Leptin Is a Growth Factor for Colonic Epithelial Cells. Gastroenterology, 121(1), 79-90.

- Javed, M., Althwanay, A., Ahsan, F., et al. (2020). Role of Vitamin D in Colorectal Cancer: A Holistic Approach and Review of the Clinical Utility. Cureus 12(9): e10734.

- Kasi, P. M., Shahjehan, F., Cochuyt, J., Li, Z., Colibaseanu, D., Merchea, A. (2018). Rising Proportion of Young Individuals With Rectal and Colon Cancer. Clin Colorectal Cancer, 18(1):e87-e95.

- Klampfer, L. (2014). Vitamin D and colon cancer. World journal of gastrointestinal oncology, 6(11), 430-437.

- Koda, M., Sulkowska, M., Kanczuga-Koda, L., et al. (2007). Expression of the obesity hormone leptin and its receptor correlates with hypoxiainducible factor-1 alpha in human colorectal cancer. Ann Oncol, 18 Suppl 6:vi116-9.

- Maalmi, H., Walter, V., Jansen, L., Boakye, D., Schöttker, B., Hoffmeister, M., Brenner, H. (2018). Association between Blood 25Hydroxyvitamin D Levels and Survival in Colorectal Cancer Patients: An Updated Systematic Review and Meta-Analysis. Nutrients, 10(7), 896.

- Maalmi, H., Walter, V., Jansen, L., Boakye, D., Schöttker, B., Hoffmeister, M., Brenner, H. (2018). Association between Blood 25Hydroxyvitamin D Levels and Survival in Colorectal Cancer Patients: An Updated Systematic Review and Meta-Analysis. Nutrients, 10(7), 896. 
- Mhaidat, N. M., Alzoubi, K. H., Kubas, M. A., Banihani, M. N., Hamdan, N., Al-Jaberi, T. M. (2021). High levels of leptin and non-high molecular weight-adiponectin in patients with colorectal cancer: Association with chemotherapy and common genetic polymorphisms. Biomedical reports, 14(1), 13.

- $\quad$ Ning, S., Wei, W., Li, J., et al. (2018). Clinical significance and diagnostic capacity of serum TK1, CEA, CA 19-9 and CA 72-4 levels in gastric and colorectal cancer patients. J Cancer, 9(3):494-501.

- Padidar, S., Farquharson, A. J., Williams, L. M., Kelaiditi, E., Hoggard, N., Arthur, J. R., Drew, J. E. (2011). Leptin up-regulates pro-inflammatory cytokines in discrete cells within mouse colon. Journal of Cellular Physiology, 226(8), 2123 2130 .

- Pludowski, P., Holick, M. F., Grant, W. B., et al. (2018). Vitamin D supplementation guidelines. The Journal of Steroid Biochemistry and Molecular Biology, 175, 125-135.

- Siegel, R., DeSantis, C., Jemal, A. (2014). Colorectal cancer statistics, 2014. CA: A Cancer Journal for Clinicians, 64(2), 104-117.

- Sun, F., Tan, Y. A., Gao, Q. F., et al. (2019). Circulating fibrinogen to pre-albumin ratio is a promising biomarker for diagnosis of colorectal cancer. J Clin Lab Anal, 33(1):e22635.

- Tojek, K., Anaszewicz, M., Szukay, B., et al. (2019). Circulating Leptin, Adiponectin, and Tumor Necrosis Factor-Alpha in Patients Undergoing Surgery Due to Colorectal Cancer. Digestion, 1-10.

- Van Harten-Gerritsen, A. S., Balvers, M. G. J., Witkamp, R. F., Kampman, E., van Duijnhoven, F. J. B. (2015). Vitamin D, Inflammation, and Colorectal Cancer Progression: A Review of Mechanistic Studies and Future Directions for Epidemiological Studies. Cancer Epidemiology Biomarkers \& Prevention, 24(12), 1820-1828.

- Wolf, A. M. D., Fontham, E. T. H., Church, T. R., et al. (2018). Colorectal cancer screening for average-risk adults: 2018 guideline update from the American Cancer Society. CA: A Cancer Journal for Clinicians, 68(4), 250-281.

- Wu, G., Xue, M., Zhao, Y., Han, Y., Zhang, S., Zhang, J., Li, C., Xu, J. (2020). Low circulating 25-hydroxyvitamin $\mathrm{D}$ level is associated with increased colorectal cancer mortality: a systematic review and dose-response metaanalysis. Bioscience reports, $40(7)$, BSR20201008. 